were significantly higher in HFD $(0.2 \pm 0.16, \mathrm{n}=7)$ compared to ND $(5.2 \pm 0.98$, $n=8$ ). No difference was found in CIA-severity between HFD and ND. However, $\mathrm{CIA}$ induction increased the number of CLS in HFD $(2.77 \pm 1.07, \mathrm{n}=6)$ and interestingly in ND animals $(8.14 \pm 0.23, n=5)$ compared to healthy ND $(0.45 \pm$ $0.03, n=4)$ and healthy HFD mice $(2.57 \pm 0.53, n=4)$ without CIA. As expected, HFD led to a significant increase in systemic leptin in healthy animals in both models. Interestingly, CIA and DMM induction decreased systemic leptin levels significantly in ND and HFD, which was more prominent in CIA. The systemic effect was not reflected by local leptin distribution in the joints $(\mathrm{CIA})$ which were not altered by diet. 5 . and 7 weeks after CIA induction HFD led to a reduction in local adiponectin and visfatin expression, which were not reflected in systemic levels.

Conclusion: The data show that HFD deteriorates OA, which is similar to observations in humans. In contrast, HFD induction showed no significant difference in $\mathrm{CIA}$ severity compared to ND. Furthermore, CIA reduced local adipokine expression under HFD at later time points but not under ND. According to high numbers of CLS in ND/CIA animals and the strong reduction of leptin in CIA with HFD, $\mathrm{CIA}$ onset and severity seems to be obesity independent and more dependent on inflammation while OA appears to be directly influenced by obesity. However, HFD-induced obesity seems to alter local and systemic adipokine expression also in CIA. Interestingly, local adipokine distribution in affected joints was independent from systemic adipokine levels.

Disclosure of Interests: Hani Manfred Sauermilch: None declared, Marie-Lisa Hülser: None declared, Carina Schreiyäck: None declared, Yubin Luo: None declared, Aline Bozec: None declared, Georg Schett Speakers bureau: AbbVie, BMS, Celgene, Janssen, Eli Lilly, Novartis, Roche and UCB, Ulf Müller-Ladner Speakers bureau: Biogen, Elena Neumann: None declared

DOI: 10.1136/annrheumdis-2020-eular.2898

\section{THU0069 MIMICKING ARTHRITIS IN VITRO TO TEST DIFFERENT TREATMENT APPROACHES}

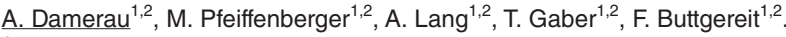
${ }^{1}$ Charité - Universitätsmedizin Berlin, Corporate Member of Freie Universität Berlin, Humboldt-Universität zu Berlin, and Berlin Institute of Health, Department of Rheumatology and Clinical Immunology, Berlin, Germany; ${ }^{2}$ German Rheumatism Research Centre (DRFZ) Berlin, a Leibniz Institute, Berlin, Germany, Berlin, Germany

Background: Our ultimate goal is to study potential drug candidates in an experimental setting of arthritis. Therefore, we aim to develop a valid human in vitro 3D joint model mimicking features of joint inflammation by applying inflammatory conditions namely immune cells and pro-inflammatory cytokines. Our in vitro 3D joint model consists of different components including an osteogenic and chondrogenic part, the joint space filled with synovial fluid, and the synovial membrane. Developed as an alternative experimental setup to animal experiments, our 3D joint model will enable us to study efficiently the effects of potential drug candidates in a human-based in vitro model.

Objectives: Here, we aimed to demonstrate the suitability of our human-based in vitro 3D osteochondral model by analyzing the influence of the main cytokines involved in the pathogenesis of RA as well as the impact of a specific therapeutic intervention.

Methods: Based on human bone marrow-derived mesenchymal stromal cells (hMSCs), we developed 3D bone and cartilage tissue components that were characterized in detail (e.g. cell vitality, morphology, structural integrity) using histological, biochemical and molecular biological methods as well as $\mu C T$ and scanning electron microscope (SEM). In brief, to establish the osteogenic component, we populated $\beta$-tricalcium phosphate (TCP) - mimicking the mineral bony part - with hMSCs, while the scaffold-free cartilage component was generated by cellular self-assembly and intermittent mechanical stimulation (fzmb $\mathrm{GmbH}$ ). Subsequently, we co-cultivated both tissue components for three weeks to generate an interconnected 3D osteochondral model. To test the suitability, we applied a cocktail of TNFa, IL-6 and MIF using concentrations reported from RA synovial fluid alone or in combination with specific therapeutic drugs and analyzed their impact by qPCR.

Results: We verified the osteogenic phenotype of our 3D bone tissue component by demonstrating an increase in mineralized bone volume and the induction of bone-related gene expression (RUNX2, SPP1 and COL1A1) as compared to the corresponding control. Secondly, we verified the chondrogenic phenotype of our cartilage tissue component by $\mathrm{HE}$ and Alcian Blue staining as well as by the reduced expression of COL1A1 and an abundant expression of COL2A1. Interestingly, co-cultivation of both components for up to 3 weeks demonstrated colonization, connectivity and initial calcification implying a transitional bridging area. Cytokine stimulation with a cocktail of TNF, IL- 6 and MIF leads to an upregulation of the metabolic marker LDHA and the angiogenic marker VEGF in both bone and cartilage. The inflammation markers IL8 and TNF are also upregulated in both components, while IL6 is downregulated in bone compared to the unstimulated control. In addition, a cytokine-induced upregulation of matrix-metalloproteases was observed especially in the cartilage component. All these cytokine-related effects could be antagonized with a cocktail of therapeutics (milatuzumab, adalimumab and tocilizumab).

Conclusion: The results of our study showed cytokine related effects of both tissue components, which can be therapeutically antagonized. By combining the components in a 96 well format, we aim to provide a mid-throughput system for preclinical drug testing.

Acknowledgments: This project is funded by the Federal Ministery of Education and Research (BMBF)

Disclosure of Interests: Alexandra Damerau: None declared, Moritz Pfeiffenberger: None declared, Annemarie Lang: None declared, Timo Gaber: None declared, Frank Buttgereit Grant/research support from: Amgen, BMS, Celgene Generic Assays, GSK, Hexal, Horizon, Lilly, medac, Mundipharma, Novartis, Pfizer, Roche, and Sanofi.

DOI: 10.1136/annrheumdis-2020-eular.3051

\section{THU0070 \\ DEFINING SYNOVIAL SIGNATURES IN THE RAT CIA MODEL: WHAT CAN WE LEARN ABOUT RA PROGRESSION?}

D. Ruelas ${ }^{1}$, R. LI ${ }^{1}$, C. Franci ${ }^{1}$, V. Lira ${ }^{1}$, D. Lopez ${ }^{1}$, L. LI ${ }^{1}$, G. Min-Oo ${ }^{1}$, J. A. DI Paolo ${ }^{1} .{ }^{1}$ Foster City, Foster City, United States of America

Background: Patients showing inadequate or no response to current therapies represent a key unmet need in rheumatoid arthritis (RA). To address this, novel or combination therapies are of high clinical interest. Identification of novel therapeutic targets requires a greater understanding of the pathogenic molecular drivers in the RA synovium. However, our current knowledge of human molecular patterns that emerge as a result of disease progression is complicated by patient-to-patient heterogeneity and access to synovial tissue.

Objectives: Here we use the current knowledge of human synovial heterogeneity to conduct a longitudinal study of global molecular responses in the rat collagen-induced arthritis $(\mathrm{CIA})$ model to better understand synovial biology, improve the preclinical modeling of human disease, and discover novel targets for RA.

Methods: A rat CIA model was performed as previously described. ${ }^{1}$ RNA-Seq was performed on 56 knee synovial tissues collected at multiple time points throughout the course of disease. Differential gene expression was determined at each individual time point and longitudinally with disease progression. Published human synovial datasets were used to categorize these genes into myeloid, lymphoid, fibroid, and low inflammatory signatures. ${ }^{2}$ Differentially expressed genes (DEGs) at each time point were compared to human synovial datasets of RA patients before and after treatment. In addition, we compared disease-driven genes in CIA to genes in RA patients that are unchanged following therapy to identify possible combination therapies.

Results: Disease pathology in the rat CIA natural history study progressed as expected: significant decreases were seen in body weight, as well as increases in ankle diameter, paw weight, and histopathology scores of joints in collagen-injected vs noninjected rats. There were 1900 DEGs identified between diseased and naïve rats over the course of disease, representing disease-induced gene signatures (Fig. 1). Comparing these DEGs to reported human RA synovial signatures, both the lymphoid and myeloid signatures were found to be highly upregulated. Interestingly, there were no significant DEGs representing the human fibroid and low inflammatory synovial signatures identified in the CIA rat model. This suggests that the rat CIA model most closely models RA patients with an immune synovial phenotype. In addition, we examined the overlap between disease-driven genes in CIA and genes in RA patients that are unchanged following therapy to identify signaling pathways that may be of utility in combination therapy. Of genes that were upregulated in $\mathrm{CIA}, 94 \%$ of genes that mapped to extracellular matrix-receptor pathways remained unchanged in the synovial tissue of RA patients following tocilizumab treatment.

Conclusion: Previous studies have shown that nearly $30 \%$ of treatment-naïve early RA patients exhibit a strong fibroid phenotype that correlates with less severe disease and a relatively poor response to disease-modifying anti-rheumatic drugs. ${ }^{3}$ These data indicate that the synovial biology associated with such patients (fibroid or pauci-immune) is not well captured in CIA, the most common preclinical RA model. To assess potential new therapies targeting these patients it will be necessary to develop alternative animal models with more intact fibroid signatures. In addition to these findings, we also characterized the global molecular changes that occur with disease progression in the $\mathrm{CIA}$ rat and made a comparison to RA patients on treatment, providing an overall understanding of 
disease-relevant pathways in the synovium that may point to possible combination therapies.

References:

[1] Trentham DE, et al. J Exp Med. 1977;146(3):857-868.

[2] Dennis G Jr, et al. Arthritis Res Ther. 2014;16(2):R90.

[3] Humby F, et al. Ann Rheum Dis. 2019;78(6):761-772.

Figure 1. Differentially expressed genes from collagen-injected versus naïve rat synovia tissue were identified over the course of disease and mapped to biological processes.

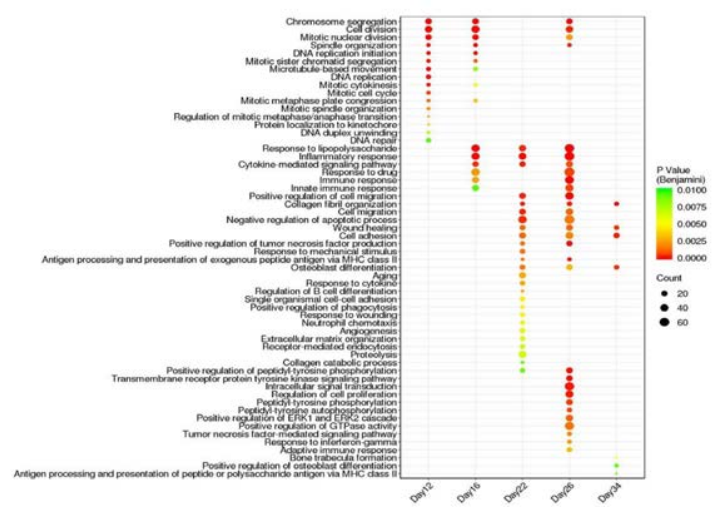

Disclosure of Interests: Debbie Ruelas Employee of: Gilead, Ruidong Li Employee of: Gilead, Christian Franci Employee of: Gilead, Victor Lira Employee of: Gilead, David Lopez Employee of: Gilead, Li Li Employee of: Gilead, Gundula Min-Oo Employee of: Gilead, Julie A. Di Paolo Employee of: Gilead DOI: 10.1136/annrheumdis-2020-eular.3695

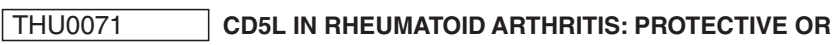 PROMOTER?}

D. Bicho ${ }^{1}$, E. Comma ${ }^{2}$, L. Oliveira ${ }^{2}$, R. F. Santos ${ }^{2}$, A. Moreira ${ }^{2}$, M. Mallo ${ }^{3}$, M. I. Bokarewa ${ }^{4}$, A. M. Carmo ${ }^{5}$ on behalf of Cell Activation and Gene Expression. ${ }^{1} 1$ I3S - Instituto de Investigação e Inovação em Saúde, Universidade do Porto, Portugal, IBMC - Instituto de Biologia Molecular e Celular, Porto, Portugal, Porto, Portugal; ${ }^{2} 1$ I3S - Instituto de Investigação e Inovação em Saúde, Universidade do Porto, Portugal, IBMC, Porto, Portugal; ${ }^{3}$ IGC - Instituto Gulbenkian de Ciência, Oeiras, Portugal; ${ }^{4}$ Department of Rheumatology and Inflammation Research, Institute of Medicine, Sahlgrenska Academy, University of Gothenburg, Sweden, Gothenburg, Sweden; ${ }^{5} 1$ I3S - Instituto de Investigação e Inovação em Saúde, Universidade do Porto, Portugal, Porto, Portugal

Background: Rheumatoid Arthritis (RA) is an aggressive auto-immune disease characterized by synovial hyperplasia and chronic inflammation. The main players of RA pathogenesis are T-cell and B-cell dependent pathways and some myeloid cells are also abundant in the synovial tissue. However, how inflammation is initiated, propagated and maintained remains controversial. Unbiased proteomic reports revealed an enrichment in the scavenger receptor CD5L, a component of serum and synovial tissues of arthritic patients. 1 Upon secretion, this blood circulating glycoprotein represses pathogenic Th17 cells, promotes M2 polarization and binds and aggregates Gram-negative and -positive bacteria.2-4 However, its mechanisms of action has not been established either in health or disease.

Objectives: We intend to clarify whether CD5L is an immune component that helps resolving RA or a factor that aggravates the disease.

Methods: We analyzed by ELISA the presence of CD5L in samples from RA patients covering different stages of the disease, and correlated with other markers of RA. In parallel, we experimentally induced collagen induced arthritis (CIA) in CD5L knockout (KO) mice to evaluate the incidence and severity of the disease. The differences between the cellular groups in circulation vs the composition on secondary lymph organs using flow cytometry were also investigated in $\mathrm{KO}$ and WT mice. The histopathology of the joints was examined, while cytokine concentrations at several timepoints and total Ig levels were measured by ELISA and cytometric bead assays, respectively.

Results: The samples from RA patients showed increased CD5L levels concomitant with the severity of the disease and a direct correlation with Sharp RTG Score or IL-6 serum levels, and inversely correlated with COMP levels. However, these correlations did not clarify whether CD5L helps to resolve RA or is a component that aggravates the disease. To clarify this aspect, we provoked CIA in CD5L KO mice and observed a higher incidence of RA, higher severity and a much lower recovery rate when compared with WT mice. To corroborate

these data, the H\&E staining of sagittal section of fore- and hindpaws revealed histopathology consistency with RA, with notable inflammatory signs especially in KO mice. WT animals with RA also showed higher levels of CD5L when compared with the control group, which confirms the observations obtained for human samples. Total serum IgG levels did not correlate with the disease severity but KO mice presented higher quantities of IgG and IL-6 when compared with WT mice.

Conclusion: Overall, these data imply that CD5L is not a promoter of the disease but rather a fundamental protective molecule against inflammation.

References:

[1] Balakrishnan L et al. (2014) Clin Proteom 11:1

[2] Wang $C$ et al. (2015) Cell 163:1413-27

[3] Sanjurjo L et al. (2018) Front Immunol 9:480

[4] Martinez VG et al. (2014) Cell Mol Immunol 11:343-354

Acknowledgments: This project has received funding from the European Union's Horizon 2020 research and innovation programme under grant agreement No683356-FOLSMART

Disclosure of Interests: None declared

DOI: 10.1136/annrheumdis-2020-eular.6376

\section{\begin{tabular}{|l|l}
\hline THU0072 THE RELATIONSHIP BETWEEN INFLAMMATION \\
\hline
\end{tabular} AND COGNITIVE IMPAIRMENT IN RHEUMATOID ARTHRITIS}

Y. H. Cheon ${ }^{1}$, H. N. Lee ${ }^{1}$, M. Kim ${ }^{1} .{ }^{1}$ Gyeongsang National University Hospital, Jin-Ju, Korea, Rep. of (South Korea)

Background: The pathophysiology of cognitive impairment remains unclear, however, several studies have demonstrated that pro-inflammatory cytokines such as Interleukin-6 (IL-6), Tumor necrosis factor-a (TNF-a) and lipocalin-2 (LCN2) are related with cognitive impairment by activation of microglia and astrocyte in brain. Rheumatoid arthritis (RA) is a representative inflammatory disease; however, the association of pro-inflammatory cytokines and LCN2 with cognitive impairment has seldomly been investigated in RA.

Objectives: Here, we determined the effect of pro-inflammatory cytokines and LCN2 on cognitive impairment in collagen-induced arthritis (CIA) mouse model. In addition, we studied the effect of TNF-a inhibitor (etanercept) on cognitive impairment.

A

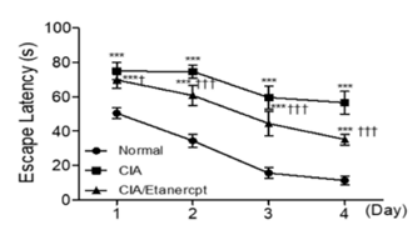

C

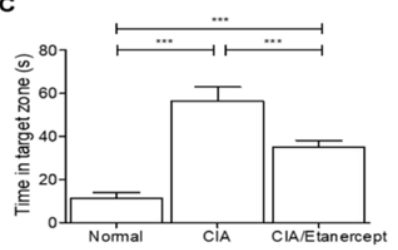

E

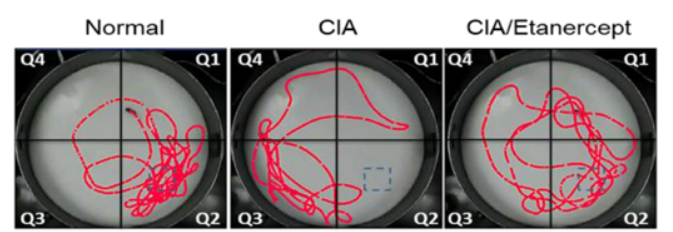

Figure 1. Comparison of learning and memory abilities in CIA mice.

For the Morris water maze, the learning phase was conducted four times per day over 5 days. (A) Escape latency to reach the platform during the training session (days 1-4), (B) escape latency to the reach the platform, (C) time spent in the target zone, (D) number of the target zone crossing and (E) tracking figures in the final trial of the last training day was also shown. Normal; Healthy group, CIA; collagen-induced arthritis group, CIA/Etanercept; collagen-induced arthritis and etanercept treatment group. Values are the means $\pm \operatorname{SEM}\left(\mathrm{n}=10\right.$ mice per group). ${ }^{*}, p<0.05,{ }^{* *} ; p<0.01,{ }^{* * *} ; p<0.001$ and $\mathrm{ns}=\mathrm{not}$ significant.
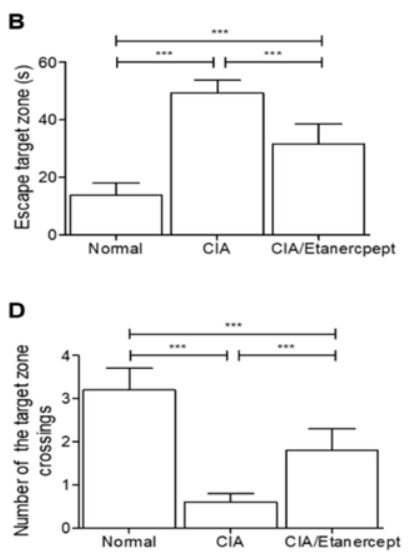

응 